\title{
The use of computer-assisted orthopedic surgery for total knee replacement in daily practice: a survey among ESSKA/SGO-SSO members
}

\author{
N. Friederich $\cdot$ R. Verdonk
}

Received: 18 November 2007 / Accepted: 26 February 2008/Published online: 26 March 2008

(C) Springer-Verlag 2008

\begin{abstract}
Computer-assisted orthopedic surgery (CAOS) for total knee arthroplasty is an emerging surgical tool, yet little is known about how it is being used in everyday orthopedic centers. We sought to better understand physicians' current practices and beliefs on this topic through performing a Web-based survey. Between December 2006 and January 2007, a 24-question survey was emailed to 3,330 members of the European Society of Sports Traumatology Knee Surgery and Arthroscopy (ESSKA) and the Swiss Orthopedic Society (SGO-SSO), with 389 (11.7\%) agreeing to participate. Of this group, 202 (51.9\%) reported that their center was equipped with a navigation system, which was an image-free based system for most (83.2\%) and was primarily used for total knee arthroplasty (61.4\%). In terms of the proportion of use, $50.5 \%$ of respondents used their navigation system in less than $25 \%$ of cases, $16.3 \%$ in $25-50 \%$ of cases, $7.4 \%$ in $51-75 \%$ of cases, and $25.7 \%$ in more than $75 \%$ of cases. The potential for improving the alignment of prosthesis was the most strongly cited reason for using a navigation system, while the potential for increasing operation times and the risk of infections were the most strongly cited reasons for not using a navigation system. Approximately half of respondents surveyed believed navigation systems were a real innovation contributing to the improvement of total knee
\end{abstract}

\footnotetext{
N. Friederich $(\bowtie)$

Department Orthopaedic Surgery and Traumatology,

Kantonsspital, 4101 Bruderholz, Basel, Switzerland

e-mail: niklaus-f.friederich@unibas.ch

R. Verdonk

Department Orthopaedic Surgery,

University Hospital Ghent, 9000 Ghent, Belgium

e-mail: rene.verdonk@rug.ac.be
}

implantation. However, heavy usage of computer-assisted navigation ( $\geq 51 \%$ of cases) was observed in only $33.1 \%$ of respondents, with only a quarter using it at rates that could be considered frequent ( $>75 \%$ of cases). Forty-eight percent of respondents said they will use a navigation system in more cases and $39.1 \%$ that their usage will stay the same. These findings indicate that CAOS is being used only moderately in current practices, though respondents generally had a positive opinion of its potential benefits. Physicians may be awaiting more data before adopting the use of these systems, though survey responses also suggest a projected increase in their use in the coming years.

Keywords Computer-assisted surgery . Surgical navigation - Orthopedic surgery · Total knee replacement $\cdot$ Survey

\section{Introduction}

Computer-assisted surgery first emerged as a clinical option in the late 1980s, when it was developed to assist neurosurgeons. Approximately a decade later, ongoing research efforts led to the development of several novel computer-assisted systems for a variety of different clinical areas, including the advent of computer-assisted orthopedic surgery (CAOS), which was primarily designed to improve the surgical treatment of orthopedic trauma and total joint arthroplasty [1].

CAOS provides physicians with patient-specific imaging data in the hopes of enhancing intra-operative guidance. Whether image-free-, fluoroscopy-, or CT-based in design, CAOS allows the surgical team to follow the changing position of both patients and relevant surgical instruments on computer monitors throughout the procedure. 
As the development of these systems has been refined over the years, they have gone from being relatively autonomous systems requiring little oversight to being highly dependent on the direct involvement of the surgeon [2]. In turn, these systems have presented new challenges to practicing orthopedic surgeons. Despite the considerable amount of attention CAOS has gained in recent years, with national orthopedic meetings often playing host to the presentation of new computer-assisted data and demonstrations of novel systems, these surgeons have not been as enthusiastic to adopt these navigational tools within their own centers. It is thought that their hesitancy is partly due to concerns over the cost (in both time and money) and ease of applying these navigational systems, although the lack of long-term data to support their proposed superiority over conventional methods is surely another major source of apprehension [1]. For these and other reasons, the use of CAOS for total knee prosthesis implantation has generated a substantial level of debate within the orthopedic community, with arguments for and against the use of these systems both easily found within the literature and elsewhere.

Opponents of CAOS often point to what they perceive as a lack of compelling data to support its use. A recently published meta-analysis surveyed 33 studies comparing navigated with conventional total knee arthroplasty (TKA) in 3,423 patients and found no evidence that CAOS improved the mean deviance from the mechanical limb axis when compared with conventional TKA [3]. Others have pointed out that even in studies in which employing CAOS for TKAs has improved the precision and accuracy of knee alignment, the skilled surgeons behind these procedures have experienced a steep learning curve and potentially prohibitive costs [4]. Many also cite the increased operative times commonly associated with CAOS as an additional deterrent to their use. A multicenter study found that patients undergoing TKA with three different navigational systems had a statistically significant increase of $16.7 \mathrm{~min}$ in surgical time when compared with those undergoing surgery with manual instrumentation [5].

A common argument among proponents, on the other hand, is that the increased risk of TKA failure associated with postoperative misalignment/malposition is significant enough to outweigh these potential limitations. Although it is estimated that approximately $90 \%$ or more of implants survive 10-15 years after surgery [6], component misalignment/malposition is thought to account for a substantial proportion of the TKAs that fail in both the early and late postoperative years [6, 7]. Even minor malpositioning/misalignment can result in early loosening, increased polyethylene wear, and decreased function [8], and existing mechanical instrumentation has been shown to result in a low rate of accuracy, thus increasing the odds of these complications occurring [9]. As these complications can be mitigated substantially by enhancing surgical precision, proponents have looked to the positive results [5, 10-16] observed with several CAOS systems as the means with which to avoid unnecessary implant failures. Analyzing a small cohort of patients (28 knees) undergoing unicompartmental knee arthroplasty, a team of United Kingdom-based researchers reported that all patients operated using a novel hands-on robot assistant achieved tibiofemoral alignment in the coronal plane within $2^{\circ}$ of the planned position, compared with only $40 \%$ of those operated using conventional methods [14]. The authors behind the study hypothesized that their use of a post-operative CT protocol allowed for a more precise recording of implant positioning than afforded to similar studies employing radiological measurements.

The same multi-center trial that discovered a $16.7 \mathrm{~min}$ increase in surgical time for CAOS also found that a normal femorotibial axis was achieved in $48.1 \%$ of CAOS cases compared with $30 \%$ for conventional methods, while a varus axis occurred in $42.2 \%$ of manual cases compared with $26.9 \%$ for CAOS [5]. Notably, all the three navigation systems studied in this trial provided statistically similar results.

Although the increased surgical time associated with CAOS remains a concern, the gap between these systems and conventional methods is lessening [2]. Additionally, the learning curve associated with CAOS in the community hospital setting has been observed to be no longer than conventional TKA methods [16]. Cost analysis projections based on short-term data also indicate that, compared with conventional TKA, computer-navigated approaches would be more cost effective in the long term by reducing the rate of revisions and complications [8].

The relatively short time period since which patients have undergone TKAs with the assistance of computerized navigation inevitably means that it will be some time before high-quality long-term results are available to address these open questions. It is possible, however, to address another pertinent aspect of this topic of which little is currently known: the practices and beliefs of everyday orthopedic surgeons with regard to the use of CAOS for knee prosthesis implantation. To do so, a Web survey was sent to members of both the European Society of Sports Traumatology Knee Surgery and Arthroscopy (ESSKA) and the Swiss Orthopedic Society (SGO-SSO). The objectives of this study were to explore the installation of navigation systems as it relates to the years a respondent has been in practice, the setting of their practice, and the number of TKAs they perform annually; to better understand the situations in which CAOS is used; to clarify the basic reasons for using or not using CAOS; and to obtain an improved understanding of the global attitude towards navigation systems in general. 


\section{Materials and methods}

Between December 2006 and January 2007, a 24-question Web survey was emailed to a variety of physicians potentially involved in performing TKAs via CAOS. All invitees were members of ESSKA and/or SGO-SSO. Swiss physicians were invited to participate through an email from N. Friederich, with the remaining international surgeons invited to participate through an email from R. Verdonk.

The survey was conducted by ISMAR Healthcare (Belgium) using the European Society for Opinion and Marketing Research's guidelines ("Conducting Market and Opinion Research Using the Internet") [17].

Frequency calculations were performed for all categorical questions. In a series comprising questions 11-20 of the survey, respondents whose centers were equipped with CAOS were presented with a series of potential motives for why they are mostly using or mostly not using these navigation systems. They were asked to rate these various motives on a Likert scale of 1-9, with 1 meaning "not true at all," 5 meaning "do not know," and 9 meaning "very much true". Mean/median calculations were then performed for all questions using Likert scales (1-9).

\section{Results}

Email invitations were sent out to 3,330 surgeons, of which 2,804 (84.4\%) were deliverable. From this group, 389 (11.7\%) agreed to participate (Fig. 1).

Demographics

The largest percentage of respondents practiced in Switzerland (20.6\%), followed by Belgium (10.8\%), Germany

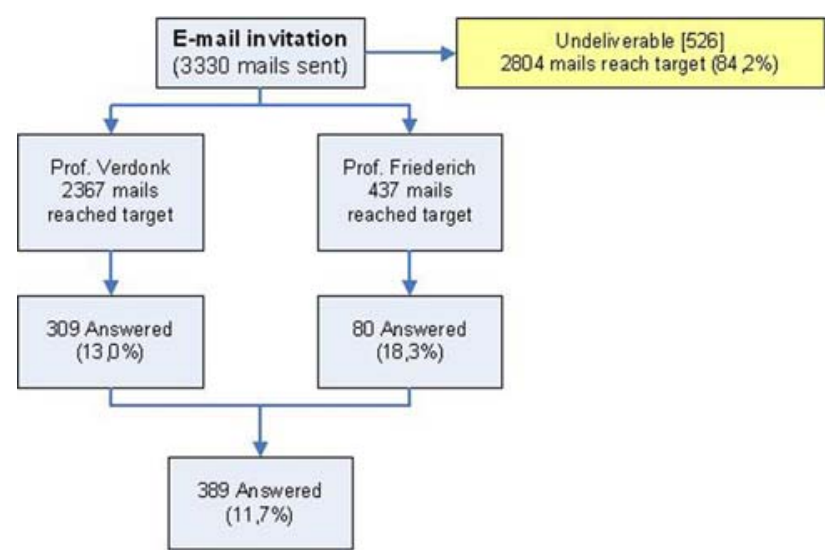

Fig. 1 Design of email survey delivery
(9.8\%), the United Kingdom (6.7\%), the United States (5.7\%), and the Netherlands (5.1\%, Fig. 2).

Eight percent of respondents had been in practice for $>30$ years, $23.4 \%$ between 21 and 30 years, 56.3\% between 6 and 20 years, and $12.3 \%$ between 1 and 5 years.

An academic/teaching hospital was the main practice setting for $48.6 \%$ of respondents, private orthopedic center for $27 \%$, non-academic/general hospital for $22.6 \%$, and "other" for $1.8 \%$.

Annually, total knee prosthesis was performed more than 100 times by $21.3 \%$ of respondents, between 51 and 100 times by $28.5 \%$, between 10 and 50 times by $40.1 \%$, and less than 10 times by $10 \%$.

Current use of navigation systems

Of the 389 total respondents, $202(51.9 \%)$ reported that their center was equipped with a navigation system. Demographic findings for these respondents are provided in Fig. 3a, d.

Of this group of 202, 83.2\% employed an image-free based navigation system in their center, $5.9 \%$ a fluoroscopy-based system, 4\% a CT-based system, and 6.9\% identified themselves as using a system categorized as "other".

When asked in which situations they use the navigation systems, $61.4 \%$ said for TKA, $49 \%$ in case of major axial deviations, $28.7 \%$ in case of minimal axial deviations, $23.8 \%$ in case of minimally invasive surgery, $22.8 \%$ in case of uni-compartmental arthroplasty, $14 \%$ for other situations, $11 \%$ never use the systems, and $2 \%$ always use the systems.

When asked whether they only use a navigation system during operation days with a small operation list, $40.1 \%$ responded that they fully disagree, $25.7 \%$ that they more or less agree, $14.9 \%$ that they more or less disagree, $12.4 \%$ that they fully agree, and $6.9 \%$ said they had no opinion.

In terms of proportion of use, $50.5 \%$ of respondents used CAOS in less than $25 \%$ of cases, $16.3 \%$ in between 25 and $50 \%$ of cases, $7.4 \%$ in $51-75 \%$ of cases, and $25.7 \%$ in more than $75 \%$ of cases. Further detail on how the proportion of use corresponds with the preceding two survey questions are provided in Fig. $4 a, b$.

Current global attitudes towards navigation systems

Mean and median results for the series of questions inquiring why respondents are mostly using $(n=67)$ or mostly not using $(n=135)$ navigation systems are provided in Figs. 5 and 6, respectively. In short, the potential for improving the alignment of a prosthesis was the most strongly cited reason for using a navigation system (8.3 mean Likert score), while the potential for increasing 
Fig. 2 Countries in which respondents practiced

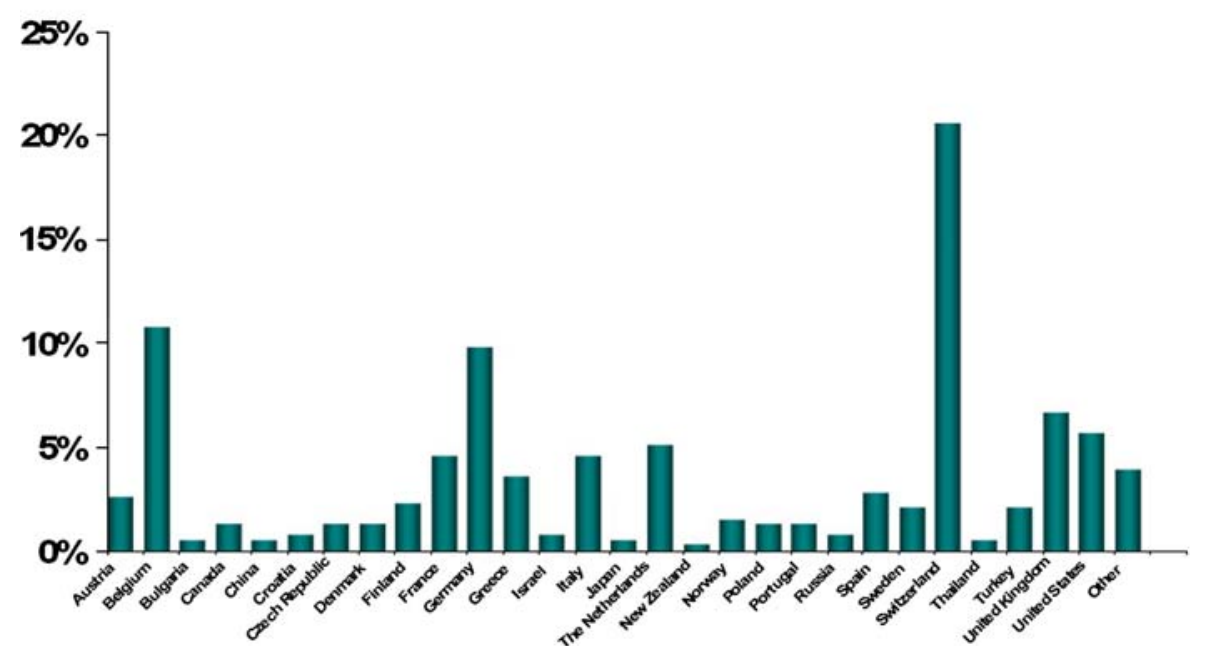

A

Is your centre equipped with a navigation system? Impact (Switzerland and Belgium)

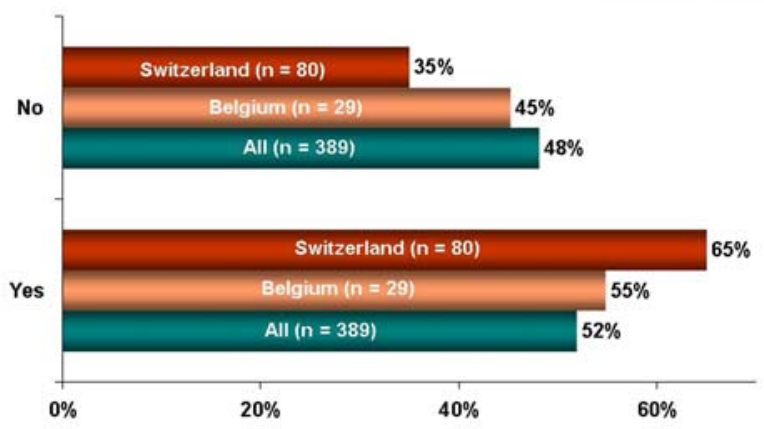

C

Is your centre equipped with a navigation system? Impact (main practice setting)

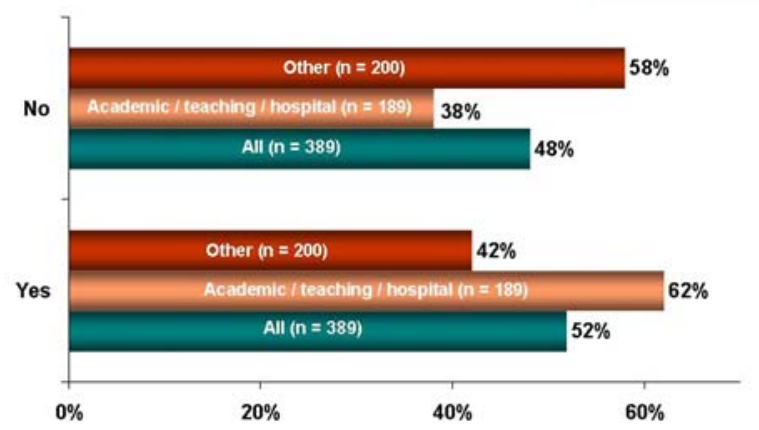

B

Is your centre equipped with a navigation system? Impact (years in practice)

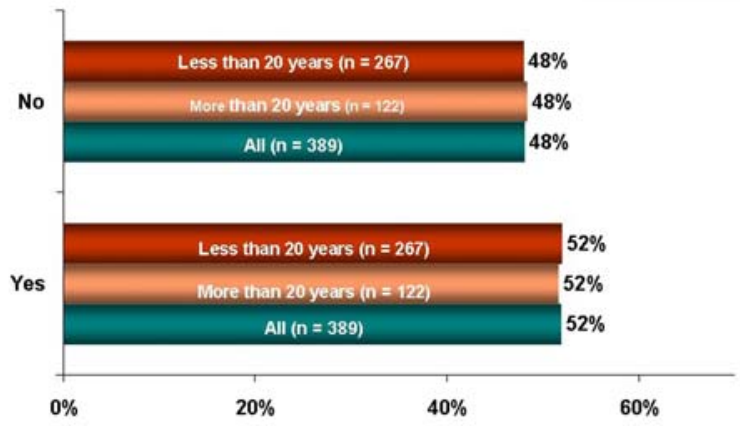

D

Is your centre equipped with a navigation system? Impact ( $\mathrm{nr}$ total knee prosthesis per YEAR)

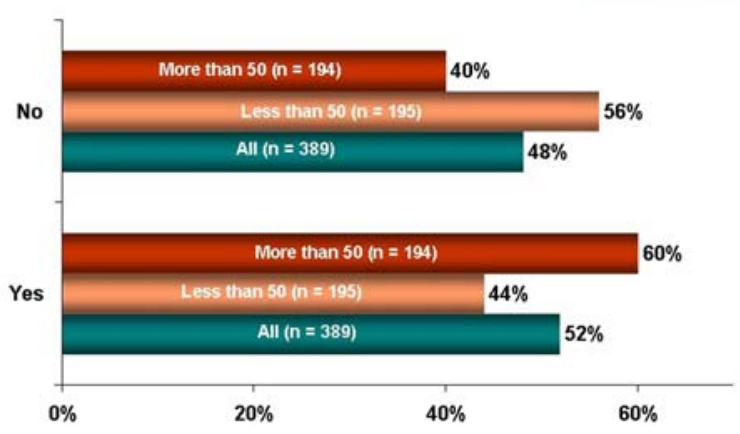

Fig. 3 Demographic characteristics of respondents reporting a navigation system at their center

operation times and the risk of infections was the most strongly cited reason for not using a navigation system (5.8 mean Likert score).
Those who currently had navigation systems at their centers were asked for the statement that best described their general feeling towards them. Of the 202 respondents, 
Fig. 4 Proportion of use based on situations in which navigation is used (a) and the use of a navigation system during operation days with a small operation list (b)
A In which situations do you use the navigation system? (users level)

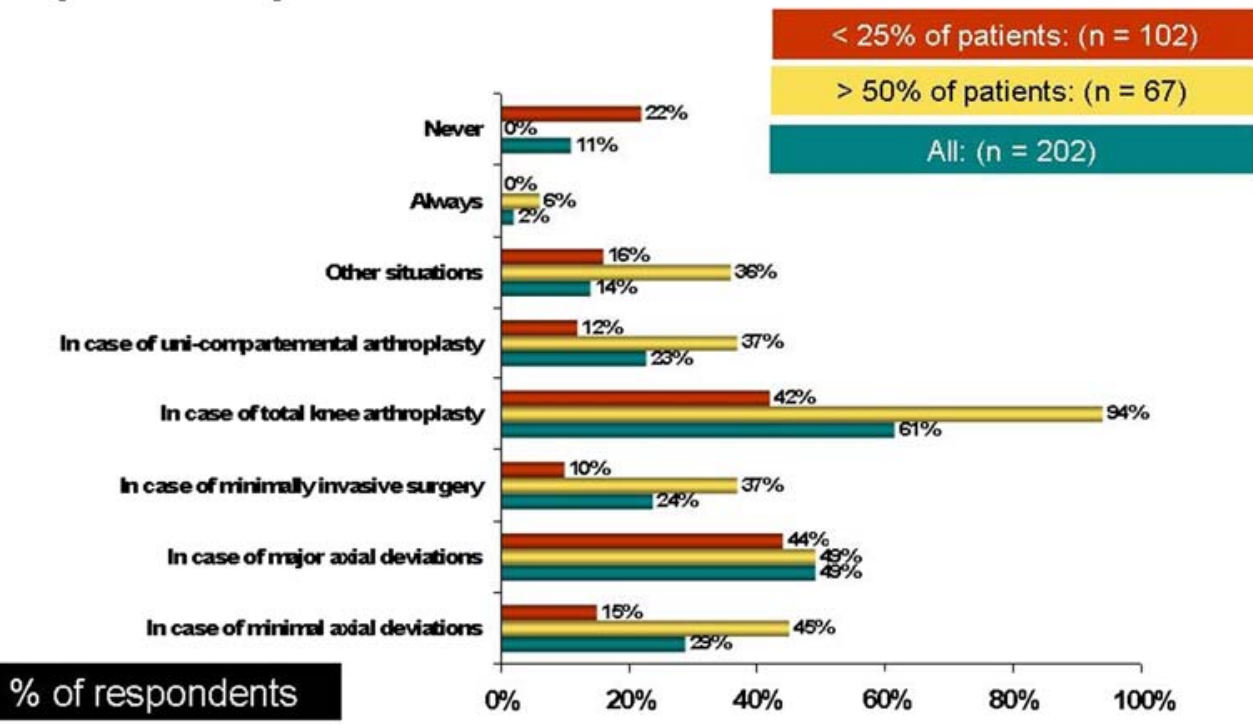

B I only use a navigation system during operation days with a small operation list (users level)

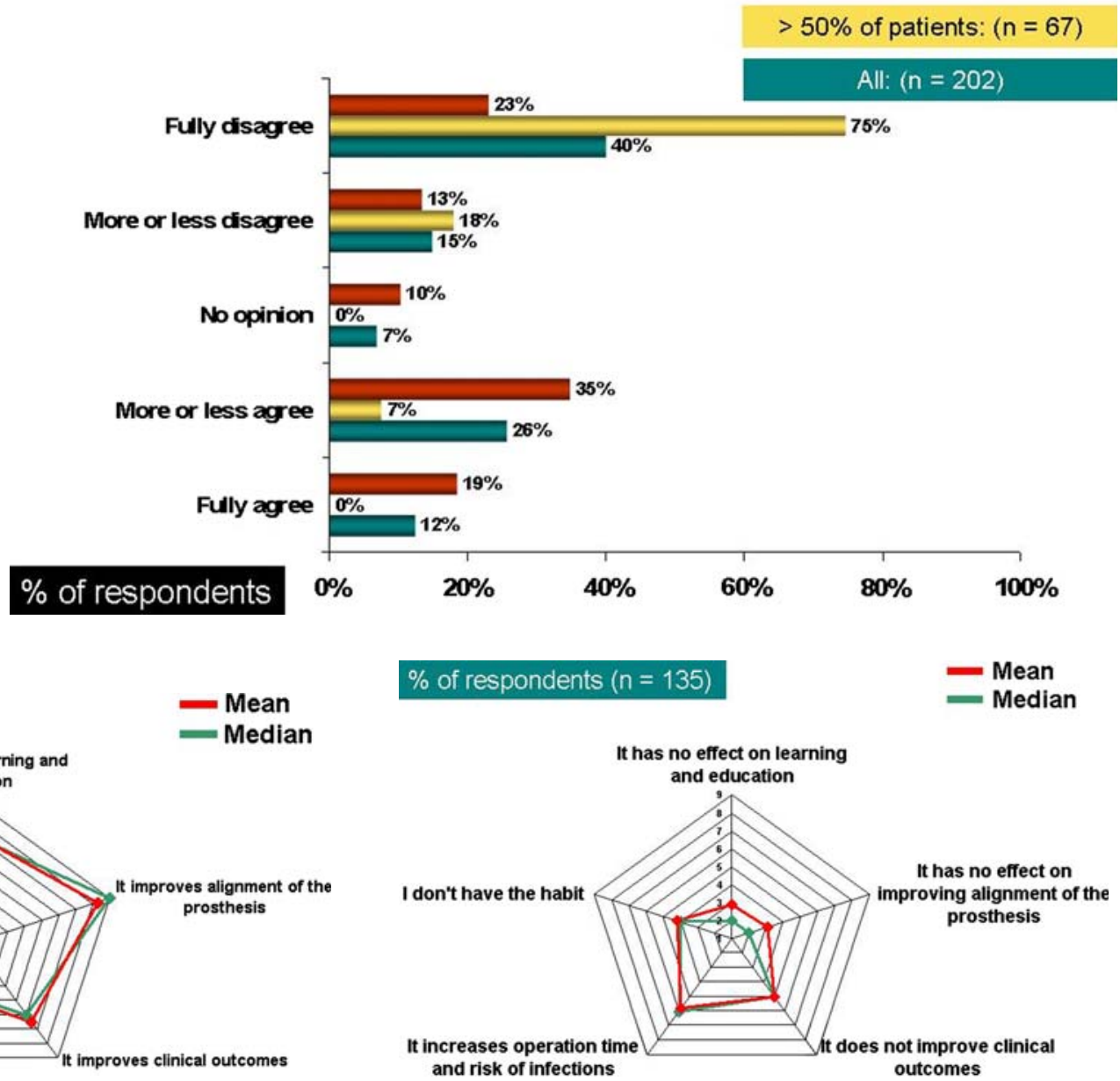

Fig. 6 Likert scale data for the cohort of infrequent users $(<25 \%$ of

\begin{abstract}
cases) on why they are (mostly) not using a navigation system
\end{abstract}
Fig. 5 Likert scale data for the cohort of heavy users $(\geq 51 \%$ of cases) on why they are (mostly) using a navigation system 
$50.5 \%$ said it was "a real innovation contributing to improvement of total knee implantation", $29.7 \%$ said that it was a "nice tool, but its value is limited", $16.8 \%$ said "its value still needs to be shown and is not visible today", and $3 \%$ said it's "fancy marketing". None of the respondents answered "no opinion".

Potential future use of navigation systems

When these 202 respondents were asked what they expect to occur in the near future, $48 \%$ said they will use a navigation system in more cases, $39.1 \%$ that their usage of the navigation system will stay the same, $6.9 \%$ said they will use a navigation system in less cases, and $5.9 \%$ did not have an opinion.

The 187 respondents who currently did not have navigation systems at their centers were asked if their centers were considering acquiring one. Of this group, $44.4 \%$ said no, $37.4 \%$ said yes, and $18.2 \%$ were not sure.

\section{Discussion}

Computer-assisted navigation for TKA has gained considerable attention since its initial development in the late 1990s but little is known about how it is being used in everyday orthopedic centers. We sought to better understand physicians' current practices and beliefs on this topic through the development of a Web-based survey.

Our study is limited by the sample we employed, making it difficult to draw any specific conclusions regarding international CAOS use. A potential response bias was created by the overrepresentation of physicians from Switzerland (20.6\% of respondents) and Belgium $(10.8 \%)$, as well as those practicing within an academic/ teaching hospital setting (48.6\%). In particular, the overrepresentation of academic centers may have in turn contributed to an overestimation of the penetration of navigation systems, which stood at $51.9 \%$ in our study. Additionally, our study is limited by the small sample size of respondents we drew from, particularly those who used navigation systems in more than $50 \%$ of patients $(33.1 \%)$, which precludes the full comparison of subgroups within the study. Although these limitations do impair our ability to provide a comprehensive analysis, the current study does offer several initial insights into the use of CAOS in TKA that may provide a valuable context with which to view data from similar future analyses.

Even though the penetration of these systems may be overestimated, CAOS does appear to have a moderate presence in contemporary orthopedic settings. Approximately half of the global respondents $(51.9 \%)$ reported that their centers are equipped with navigation systems, of which image-free based systems accounted for an overwhelming majority $(83.2 \%)$.

As was to be expected with the aforementioned limitations in the study population, Swiss respondents and those practicing in academic centers reported the highest penetration of navigation systems (65\% and $62 \%$, respectively). Predictably, larger volume practices were more likely to employ a navigation system, with those performing more than 50 TKAs annually $(n=194)$ reporting a $60 \%$ penetration of CAOS in their centers, compared with $44 \%$ for those performing less than 50 TKAs annually $(n=195)$. The number of years a physician was in practice, however, had no significant impact on whether their center was equipped with a CAOS.

The actual use of these navigation systems when present was lower than perhaps could have been expected given the overall positive opinion respondents had towards these systems. Just over half of the respondents with CAOS at their centers $(50.5 \%)$ said that they believe these systems to be "a real innovation contributing to improvement of total knee implantation". It could be presumed that this positive opinion would translate into a somewhat equivalent proportion of usage. However, what could be defined as heavy usage ( $\geq 51 \%$ of cases) was observed in only $33.1 \%$ of respondents, with only a quarter of respondents using it at rates that could be considered frequent ( $>75 \%$ of cases).

Heavy users employed CAOS primarily for TKA (94\%), followed by cases of major $(49 \%)$ and minimal axial deviations (45\%). According to Likert scale analysis, their most strongly cited reasons for CAOS use were their belief that it improves alignment of the prosthesis, enhances learning and education, and improves clinical outcomes.

A significant majority of respondents $(66.8 \%)$ can be considered low proportion users of CAOS ( $<50 \%$ of cases). Just over half $(50.5 \%)$ use navigation at a rate that can be considered infrequent ( $<25 \%$ of cases), with $22 \%$ of these users claiming to never use navigation at all. When infrequent users employ CAOS, they do so in a wide variety of manners, though primarily in cases of major axial deviations $(44 \%)$ or for TKA $(42 \%)$. Low proportion users are more likely to only use navigation systems on days without a large operation list $(34.8 \%$ more or less agreed with this statement; $18.5 \%$ fully agreed). Though unlikely to believe that CAOS has no effect on learning or education (2.9 mean Likert score) or on improving prosthesis alignment (3.1), their doubt surrounding these systems increases when asked if they do not improve clinical outcomes (5) or if they increase operation time and the risk of infections (5.8). However, as the Likert scores on these last two measures attest, their level of doubt is somewhat insignificant.

Perhaps a more reasonable conclusion regarding the relatively low rate of CAOS use among respondents is that 
their reluctance is primarily a response to the perceived lack of quality data to support it. When asked for their general feelings regarding navigation systems, $29.7 \%$ of respondents with systems at their centers said it was a "nice tool, but its value was limited" and $16.8 \%$ said "its value still needs to be shown and is not visible today". This could be a reflection of studies that question the superiority of CAOS, such as a recent meta-analysis that found little significant benefit when these systems were compared with conventional methods for TKA [3]. In this analysis, navigation-assisted surgery only reduced the risk of $3^{\circ}$ of malalignment by $25 \%$, avoiding unfavorable component positioning in 1 out of 5 patients. Though this meta-analysis was potentially limited by the use of the mechanical axis as a marker of alignment and the general lack of methodological standards among the studies chosen, it is nonetheless indicative of the clinical doubts surrounding the use of CAOS for TKA.

Another criticism often directed at CAOS for TKA is that its use is driven by marketing efforts from device companies, which in turn may be reflected in increased patient demand. Interestingly, we found that only a minority of respondents (3\%) believed navigation systems were simply the result of "fancy marketing". Additionally, patient demands/expectations were the least strongly cited reason for employing CAOS among heavy users.

Although some question whether large variations in implant alignment necessarily translate into an increased failure rate at mid- and long-term follow-up points [4], it is generally accepted that proper implant positioning contributes significantly to the success of TKA [18]. Several studies have reported positive initial results regarding implant position [5, 10-16] and other clinical endpoints, such as reductions in blood loss [15, 19] and emboli [20], when CAOS is compared with conventional methods of TKA. Recently, encouraging results have also been observed when CAOS is used for performing revision TKA's [21]. As we are in the early stages of popular use of CAOS, data are unavailable to shed light on whether these improvements will yield long-term benefits in clinical outcomes.

As physician experience with these systems increases, so too will the quality of the long-term outcomes data we will have with their use, which in turn will give clinicians the means to make more informed choices regarding CAOS. In our cohort of surgeons, $37.4 \%$ said their center is considering acquiring such a system. Additionally, $48 \%$ of respondents who currently have navigation systems at their centers said they expect to employ it in more cases in the near future.

Continued monitoring of physician beliefs and experiences with these systems will give us a better perspective of their "real world" applicability as we await long-term clinical data. Surveys of the nature conducted here, though larger in scale to facilitate enhanced data procurement, are warranted.

Acknowledgments An unconditional grant was received by Plus Orthopedics AG, Rotkreuz, Switzerland. The authors thank Peter Fennema (Rüschlikon, Switzerland), Louis Smets (Ismar Healthcare; Lier, Belgium), and John Watson (New York, USA) for their input. The offices of ESSKA and SGO-SSO assisted us in contacting their respective members.

\section{References}

1. Kahler DM (2004) Image guidance: fluoroscopic navigation. Clin Orthop Relat Res 421:70-76

2. Davies BL, Rodriguez y Baena FM, Barrett AR, Gomes MP, Harris SJ, Jakopec M, Cobb JP (2007) Robotic control in knee joint replacement surgery. Proc Inst Mech Eng [H] 221:71-80

3. Bauwens K, Matthes G, Wich M, Gebhard F, Hanson B, Ekkernkamp A, Stengel D (2007) Navigated total knee replacement. A meta-analysis. J Bone Joint Surg Am 89:261-269

4. Callaghan JJ, Liu SS, Warth LC (2006) Computer-assisted surgery: a wine before its time: in the affirmative. J Arthroplasty 21(4 Suppl 1):27-28

5. Macule-Beneyto F, Hernandez-Vaquero D, Segur-Vilalta JM, Colomina-Rodriguez R, Hinarejos-Gomez P, Garcia-Forcada I, Seral Garcia B (2006) Navigation in total knee arthroplasty. A multicenter study. Int Orthop 30:536-540

6. Sharkey PF, Hozack WJ, Rothman RH, Shastri S, Jacoby SM (2002) Insall Award paper. Why are total knee arthroplasties failing today? Clin Orthop Relat Res 404:7-13

7. Ritter MA, Faris PM, Keating EM, Meding JB (1994) Postoperative alignment of total knee replacement. Its effect on survival. Clin Orthop Relat Res 299:153-156

8. Dong H, Buxton M (2006) Early assessment of the likely costeffectiveness of a new technology: a Markov model with probabilistic sensitivity analysis of computer-assisted total knee replacement. Int J Technol Assess Health Care 22:191-202

9. Stulberg SD (2003) How accurate is current TKR instrumentation? Clin Orthop Relat Res 415:177-184

10. Bathis H, Perlick L, Tingart M, Lüring C, Zurakowski D, Grifka J (2004) Alignment in total knee arthroplasty. A comparison of computerassisted surgery with the conventional technique. J Bone Joint Surg Br 86:682-687

11. Haaker RG, Stockheim M, Kamp M, Proff G, Breitenfelder J, Ottersbach A (2005) Computer-assisted navigation increases precision of component placement in total knee arthroplasty. Clin Orthop Relat Res 433:152-159

12. Victor J, Hoste D (2004) Image-based computer-assisted total knee arthroplasty leads to lower variability in coronal alignment. Clin Orthop Relat Res 428:131-139

13. Picard F, Deakin AH, Clarke JV, Dillon JM, Gregori A (2007) Using navigation intraoperative measurements narrows range of outcomes in TKA. Clin Orthop Relat Res 463:50-57, (Epub ahead of print)

14. Cobb J, Henckel J, Gomes P, Harris S, Jakopec M, Rodriguez F, Barrett A, Davies B (2006) Hands-on robotic unicompartmental knee replacement: a prospective, randomised controlled study of the acrobot system. J Bone Joint Surg Br 88:188-197

15. Chauhan SK, Scott RG, Breidahl W, Beaver RJ (2004) Computer-assisted knee arthroplasty versus a conventional jig-based technique. A randomised, prospective trial. J Bone Joint Surg Br 86:372-377 
16. Daubresse F, Vajeu C, Loquet J (2005) Total knee arthroplasty with conventional or navigated technique: comparison of the learning curves in a community hospital. Acta Orthop Belg 71:710-713

17. European Society for Opinion and Marketing Research. ESOMAR world research codes and guidelines: Conducting market and opinion research using the internet. Available at: http://www.esomar. org/uploads/pdf/ESOMAR_Codes\&Guideline-Conducting_ research_using_Internet.pdf. Accessed on July 21, 2007

18. Siston RA, Giori NJ, Goodman SB, Delp SL (2007) Surgical navigation for total knee arthroplasty: a perspective. J Biomech 40:728-735
19. Kalairajah Y, Simpson D, Cossey AJ, Verrall GM, Spriggins AJ (2005) Blood loss after total knee replacement: effects of computer-assisted surgery. J Bone Joint Surg Br 87:1480-1482

20. Kalairajah Y, Cossey AJ, Verrall GM, Ludbrook G, Spriggins AJ (2006) Are systemic emboli reduced in computer-assisted knee surgery? A prospective, randomised, clinical trial. J Bone Joint Surg Br 88:198-202

21. Perlick L, Bathis H, Perlick C, Luring C, Tingart M, Grifka J (2005) Revision total knee arthroplasty: a comparison of postoperative leg alignment after computer-assisted implantation versus the conventional technique. Knee Surg Sports Traumatol Arthrosc 13:167-173 